\title{
Randomised, double-blind comparison of a fixed co- formulation of intra-articular polynucleotides and hyaluronic acid versus hyaluronic acid alone in the treatment of knee osteoarthritis: two-year follow-up
}

\author{
Cesare Stagni \\ Istituto Ortopedico Rizzoli \\ Martina Rocchi ( $\triangle$ martina.rocchi@ior.it ) \\ Istituto Ortopedico Rizzoli \\ Alessandro Mazzotta \\ Istituto Ortopedico Rizzoli \\ Nicolandrea Del Piccolo \\ Istituto Ortopedico Rizzoli \\ Nicola Rani \\ Istituto Ortopedico Rizzoli \\ Marco Govoni \\ Istituto Ortopedico Rizzoli \\ Leonardo Vivarelli \\ Istituto Ortopedico Rizzoli \\ Francesca Veronesi \\ Istituto Ortopedico Rizzoli \\ Michela Fini \\ Istituto Ortopedico Rizzoli \\ Dante Dallari \\ Istituto Ortopedico Rizzoli
}

\section{Research Article}

Keywords: Knee osteoarthritis, knee function, hyaluronic acid, KSS, knee pain, PN-HPT ${ }^{T M}, 71$ polynucleotides, WOMAC

Posted Date: February 17th, 2021

DOI: https://doi.org/10.21203/rs.3.rs-150017/v1 
License: (c) (i) This work is licensed under a Creative Commons Attribution 4.0 International License. Read Full License 
1 Randomised, double-blind comparison of a fixed co-formulation of intra-articular

2 polynucleotides and hyaluronic acid versus hyaluronic acid alone in the treatment

3 of knee osteoarthritis: two-year follow-up

4

5 Cesare Stagni, $\mathrm{MD}^{1}$, Martina Rocchi, $\mathrm{MD}^{1}$, Alessandro Mazzotta, $\mathrm{MD}^{1}$, Nicolandrea Del

6 Piccolo, MD ${ }^{1}$, Nicola Rani, MD ${ }^{1}$, Marco Govoni, PhD ${ }^{1}$, Leonardo Vivarelli, MEng ${ }^{1}$,

$7 \quad$ Francesca Veronesi, $\mathrm{PhD}^{2}$, Milena Fini, $\mathrm{MD}^{2}$, Dante Dallari, MD ${ }^{1}$

$8{ }^{1}$ Reconstructive Orthopaedic Surgery and Innovative Techniques -

9 Musculoskeletal Tissue Bank, IRCCS Rizzoli Orthopedic Institute, Bologna, Italy;

$10 \quad{ }^{2}$ Laboratory of Preclinical and Surgical Studies, IRCCS Rizzoli Orthopedic

11 Institute, Bologna, Italy.

14 Corresponding author: Martina Rocchi, MD, Reconstructive Orthopaedic Surgery and Innovative Techniques - Musculoskeletal Tissue Bank, IRCCS Rizzoli

16 Orthopedic Institute, Via Di Barbiano 1/10, 40136 Bologna, Italy (email:

17 martina.rocchi@ior.it)

20 Emails of other authors: Cesare Stagni (cesare.stagni@ior.it); Martina Rocchi

21 (martina.rocchi@ior.it); Alessandro Mazzotta (alessandro.mazzotta@ior.it);

22 Nicolandrea Del Piccolo (nicolandrea.delpiccolo@ior.it); Nicola Rani 
23 (nicola.rani@ior.it); Marco Govoni (marco.govoni@ior.it); Leonardo Vivarelli

24 (leonardo.vivarelli@ior.it); Francesca Veronesi (francesca.veronesi@ior.it); Milena

25 Fini (milena.fini@ior.it); Dante Dallari (dante.dallari@ior.it)

29 ABSTRACT

30 Background: A first-year interim analysis of this two-year study suggested that intra31 articular injections of highly purified, natural-origin polynucleotides and hyaluronic 32 acid (HA) as a fixed combination (PNHA) might improve knee function and joint pain 33 more effectively than HA alone in patients with knee osteoarthritis (OA). Purpose of 34 the second-year analysis herein described was verifying whether the first-year interim outcomes persist over the whole two-year period.

36 Methods: Randomised, double-blind, HA-controlled clinical trial in 100 knee OA 37 patients (98 randomised, 69 completing the study) in a high-specialisation tertiary care setting. The hypothesised difference of efficacy between PNHA and HA for the original sample size estimate is $20 \%$. Treatment cycle: 3 weekly intra-articular knee injections of either PNHA or HA. Evaluations: Western Ontario and McMaster

41 Universities (WOMAC) score and Knee Society Score (KSS) as, respectively, primary 42 and secondary endpoints, evaluated at baseline and after 2, 6, 12, and 24 months; 43 synovial fluid levels of proinflammatory mediators (biochemical and 44 immunoenzymatic assays at baseline and the end of the treatment cycle). Adverse 
effects investigated at each control visit. Statistical analysis: Kruskal-Wallis test for independent samples (nonparametric one-way analysis of variance) after correction of means for age, Body Mass Index and Kellgren-Lawrence grade. If significant, pairwise post-hoc Sidak multiple comparisons.

Results: KSS total score and KSS pain item: significant improvement in both groups, with significantly more pain improvement in patients treated with PNHA (2-point reduction) than HA (1-point reduction). Both groups experienced significant longterm reductions in WOMAC total scores: significantly stronger in PNHA-treated patients after 24 months with a steady difference of $16 \%$ favouring PNHA in WOMAC pain subscore. No clinically significant adverse events in either group.

Conclusions: The outcomes of the 2-year study confirmed that a short cycle of intraarticular treatment ( 3 weekly double-blind injections) with polynucleotides (longacting viscosupplementation properties, pro-trophic activity on chondrocytes, painrelieving properties) in fixed combination with high molecular weight hyaluronic acid is more effective in improving knee function and pain in knee OA patients than HA alone. PNHA may be elective for viscosupplementation in knee OA patients with fastidious and resistant pain, signs of inflammation or worsening disease.

3 Trial Registration (ClinicalTrials.gov database Identifier): NCT02417610

4 Registration, 15/04/2015

65 ClinicalTrials.gov database link:

6 https://clinicaltrials.gov/ct2/show/NCT02417610?term=NCT02417610\&cntry=IT\&dra $7 \mathrm{w}=2 \& \mathrm{rank}=1$ 


\section{KEYWORDS}

70 Knee osteoarthritis; knee function; hyaluronic acid; KSS; knee pain; PN-HPT ${ }^{\mathrm{TM}}$; 71 polynucleotides; WOMAC

72

BACKGROUND

76 The debate about the real value of hyaluronic acid (HA) as infiltrative therapy of knee 77 osteoarthritis (OA) is far from over in evidence-based guidelines and consensus 78 reports. ${ }^{1-3}$ Highly purified polynucleotides from trout gonads, also known with the 79 acronym PN-HPT ${ }^{\mathrm{TM}}$ (Polynucleotides Highly Purified Technology), provide 80 persistent viscosupplementation, show trophic properties on chondrocytes and 81 mesenchymal cells, and reduce pain more effectively and more rapidly than HA.4-6 In82 vitro and in-vivo synergy between $\mathrm{PN}-\mathrm{HPT}^{\mathrm{TM}}$ and HA on chondrocyte trophism and 83 pain control has also been convincingly established - a strong rationale to administer 84 the two viscosupplementation agents together. ${ }^{7}$

85 The study aimed to verify over two years whether "the association of PN-HPT" and 86 HA injections would reduce pain in patients affected by knee OA more than HA 87 alone", and whether "it is more effective in improving knee function and pain, in 88 joints affected by OA, compared with HA alone", as suggested by the authors in their 89 first-year interim report. ${ }^{8}$ Analysing the final two-year outcomes of the study also 90 aimed to verify whether the clinical synergy between PN-HPT ${ }^{\mathrm{TM}}$ and HA, which the 
91 first-year interim analysis suggested, is persistent over a much longer time or it is just 92 a transient medium-term effect.

94

95 METHODS

96 Study Design, Sample Size Estimate and Patient Selection

97 A hundred knee OA patients, aged between 51and 74 years, were initially screened 98 between mid-September 2014 and mid-July 2015, and 98 randomised, in a double99 blind, single-centre, HA-controlled study. Two patients were excluded after failure to 100 meet the inclusion criteria. The authors carried out the study at the Rizzoli Orthopedic 101 Institute, Bologna, Italy, in rigid agreement with the most recent clinical practice 102 guidelines and ethical regulations (for details, see the report that discussed the interim 103 outcomes after the first year of treatment). ${ }^{8}$ The final, two-year outcomes are herein 104 illustrated. Demographics and the initially randomised knee OA patients' selection 105 criteria are summarised in Table 1 and Table 2, respectively. The intake of NSAIDs 106 and other drugs was free during the two-year study period; investigators only 107 recorded the NSAIDs consumption since the last visit.

108 The assumptions initially leading to the sample size calculation and the technicalities 109 adopted for creating the randomisation list and preserving the double-blindness all 110 those involved, patients, investigators, data collectors and outcome assessors, were 111 exhaustively described in the first-year interim report. ${ }^{8}$ 
112 The main points about the sample size estimate are herein summarised. With the per 113 cent WOMAC change at 12 months considered as the primary endpoint, the following 114 formula gave an estimate of the needed sample size: ${ }^{8}$

115

12-month WOMAC - baseline WOMAC $\Delta$ WOMAC (per cent difference vs baseline) $=$ 100 - baseline WOMAC

120 Based on previous HA literature and exploratory unpublished PNHA little studies,

121 the basic assumption leading to the original sample size estimate was that standard 122 deviations were $26.9 \%$ for PNHA-treated patients and 39.1\% for HA-treated patients.

123 Further assumptions were that standard deviations would be similar for the two

124 populations to be enrolled. The two intra-articular treatments would differ by at least $12520 \%$, in terms of clinical efficacy, under the null hypothesis that the two treatments 126 had similar WOMAC per cent variations. With the assumption of a false-positive 127 (alpha) error of 0.05 and power to avoid false negatives of at least 0.80 , a minimum 128 clinically meaningful difference of $20 \%$ and a drop-out rate of $10 \%$, the minimum 129 estimated number of patients was 50 per group (100 overall). ${ }^{8}$

130 The coded packages of PNHA and HA syringes were identical with syringes masked 131 by identical sleeves. The randomisation list reported the numerical code on syringe 132 packages; investigators received the randomisation codes for each patient sealed in an 133 envelope. ${ }^{8}$

134 Ninety out of initially randomised patients completed the study at [T5] (interim 135 evaluation after the first year of treatment), 46 in the PNPHA study group and 44 in 
136 the HA control group; all of them then progressed to [T6] (end of study). Sixty-nine 137 patients completed the 2-year study (final follow-ups: 70\%). All the patients who had 138 dropped out at the end of the first year did it for personal reasons. ${ }^{8}$

139

\begin{tabular}{|c|c|c|c|}
\hline & $\begin{array}{l}\text { All patients } \\
(\mathrm{n}=100)\end{array}$ & $\begin{array}{l}\text { Study Group } \\
\text { (PNHA, n=49 }\end{array}$ & $\begin{array}{l}\text { Control Group } \\
(\mathrm{HA}, \mathrm{n}=49)\end{array}$ \\
\hline Age, yrs & $50-75(63.8 \pm 5.8)$ & $63.4 \pm 6.5$ & $64.2 \pm 5.1$ \\
\hline Kellgren-Lawrence grade ${ }^{9}$ & $2 \pm 0.7$ & $1.9 \pm 0.6$ & $2.1 \pm 0.7$ \\
\hline Sex, male/female, $n$ & $46 / 54$ & $24 / 26$ & $22 / 28$ \\
\hline Body Mass Index, kg/m² & $28,1 \pm 3,5$ & $28,1 \pm 3,4$ & $28,1 \pm 3,7$ \\
\hline Weight, kg & $80.0 \pm 11.6$ & $80.2 \pm 10.2$ & $79.8 \pm 13$ \\
\hline Height, cm & $168,5 \pm 9.2$ & $168,9 \pm 9.5$ & $168,1 \pm 9.0$ \\
\hline
\end{tabular}

140 Table 1 Demographics of the originally screened knee osteoarthritis patients. ${ }^{8,9}$

\title{
Exclusion criteria
}

\begin{abstract}
Abuse of alcohol or drugs
Pregnancy or breastfeeding

Patients who underwent repeated infiltrative therapies or patients who only underwent a single HA infiltration cycle, although performed less than six months before enrolment Ongoing treatment with systemic anticoagulants or steroids, or therapy suspended for less than one month

Hypersensitivity to the study products, previous bone fractures, severe knee trauma, joint deformities, rheumatoid arthritis, inflammatory diseases of joints, previous surgical procedures (e.g., meniscectomy, scope debridement)

Haematological diseases or local skin lesions in the site of treatment inoculation
\end{abstract}

143 Table 2 The criteria adopted for selecting the 98 enrolled patients. ${ }^{8}$ 
147 The regulatory classification of the patented, proprietary fixed PNHA combination

148 investigated in the 2-year study was as a Class-III CE-marked (0373) medical device:

149 pre-filled, single-use, neutral glass 2-mL syringes dosed at $10 \mathrm{mg} / \mathrm{mL}$ of natural-origin

$150 \mathrm{PN}-\mathrm{HPT}^{\mathrm{TM}}$ and $10 \mathrm{mg} / \mathrm{mL}$ of a biotechnological sodium HA (molecular weight > 1500

$151 \mathrm{kDa}$ ) for an overall syringe content of $40 \mathrm{mg}$ in $2 \mathrm{~mL}$ of active principles. The European

152 Union's regulatory authorities and several extra-European countries registered the

153 proprietary fixed PNHA combination (brand, POLIART ${ }^{\circledR}$, Mastelli Srl, San Remo,

154 Italy) for the indication "intra-articular treatment of degenerative chondral disorders".

155 The control HA product (IALART ${ }^{\circledR}$, Mastelli Srl, San Remo, Italy), is also a Class III

156 CE 0373 commercially available medical device of HA (1200-1500 kDa), industrially

157 obtained from bacterial fermentation and dosed at $40 \mathrm{mg}$ in $2 \mathrm{~mL}$. The formulation of

158 both study products was as absorbable, viscoelastic sterile gels.

159 Highly skilled specialists performed three weekly intra-articular double-blind 160 infiltrations with 18 to $22 \mathrm{G}$ needles at baseline [T0] and over the following two weeks

161 [T1] and [T2], under aseptic conditions and following standard intra-articular

162 techniques (injected amount at each session, $2 \mathrm{~mL}$ ). Samples of the synovial fluid

163 (nearly $6 \mathrm{~mL}$ of the removed excess synovial fluid) were collected and sent to the

164 laboratory before the first infiltration [T0] and at the end of the treatment cycle [T2]

165 (Figure 1). 


\begin{tabular}{|c|c|c|}
\hline \begin{tabular}{c|c|} 
T0: \\
$1^{\text {st }}$
\end{tabular} & $\begin{array}{c}\text { T1: } \\
2^{\text {nd }}\end{array}$ & $\begin{array}{c}\text { T2: } \\
3^{\text {rd }} \\
\text { injection }\end{array}$ \\
injection & injection \\
\hline
\end{tabular}
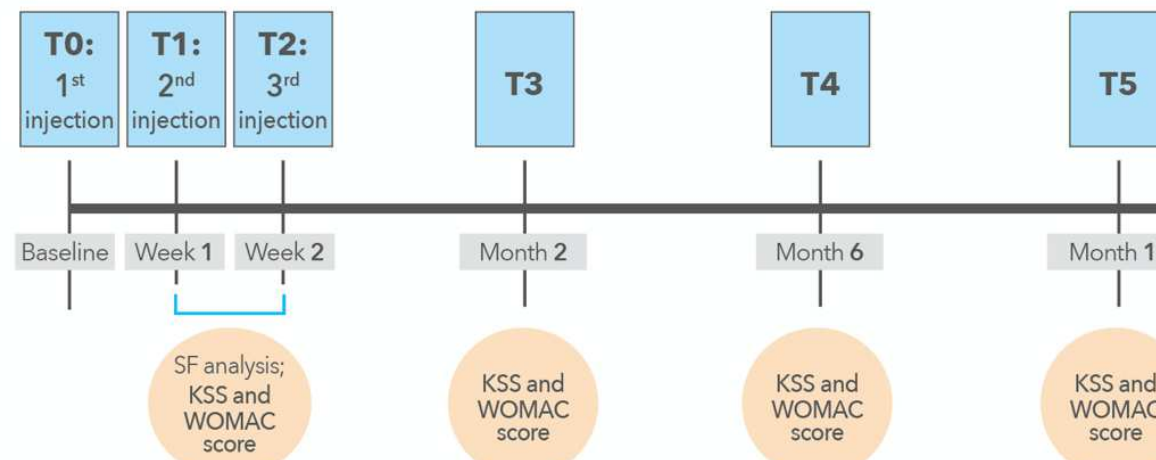

T6

174

175 Figure 1 [T0] to [T2]: timing of the three double-blind PNHA and HA intra-articular

176 injections and assessments planned over the first two study weeks (synovial fluid analysis

177 and first KSS and WOMAC evaluation); [T3] to [T6]: timing of the KSS and WOMAC

178 evaluations planned over the residual 2-year study period.

179

180

181 Follow-Up Assessments

182 The knee joint function and pain were assessed, with the help of the Knee Society

183 Score $(\mathrm{KSS})^{10}$ and the self-administered Western Ontario and McMaster Universities

184 (WOMAC) score ${ }^{11}$, at baseline [T0] and after 2 [T3], 6 [T4], 12 [T5] and 24 months [T6]

185 during the 2-year follow-up. A radiographic examination complemented the final

186 clinical evaluation at [T6]. The WOMAC pain subscore was the primary endpoint; KSS,

187 the overall WOMAC score and NSAID consumption were secondary endpoints.

188 Assays of the viscosity of the synovial fluid and the synovial fluid levels of several

189 inflammatory markers - matrix metalloproteinase-1 (MMP1), MM13, tissue inhibitor 
of MMP1 (TIMP1), interleukins 1ß (IL-1ß) and IL-6, Tumor Necrosis Factor- $\alpha$ (TNF- $\alpha$ ),

191 chemokine IL-8, prostaglandin $\mathrm{E}_{2}\left(\mathrm{E}_{2}\right)$ - were also planned in 40 patients. Assays

192 timing: baseline [T0] and the end of the 2-week treatment cycle [T2] using standard

193 biochemical and immunoenzymatic assays (complete technical details of commercial

194 assays and procedures are available in Ref. 8). As far as possible, all WOMAC and KSS

195 scoring, and indeed all clinical evaluations and biochemical assays on synovial fluid,

196 were performed by the same investigator with only a very few exceptions. Local or

197 systemic side effects were recorded in the electronic clinical report form at each

198 follow-up visit, and the casual relationship immediately assessed and reported for

199 further evaluation.

200

201

202 Statistical Analysis

203 Descriptive data were tabulated as means \pm standard deviations (SD) and graphically 204 as boxplots. The general linear model for repeated measures or Kruskal-Wallis test for 205 independent samples (nonparametric one-way ANOVA test) was applied, after 206 correction of means for age, Body Mass Index (BMI) and Kellgren-Lawrence (KL) 207 grade, ${ }^{12}$ to assess for the effect of treatments on the follow-up curves. Using the 208 nonparametric one-way ANOVA test was justified because data (WOMAC, KSS, KSS 209 subscore for pain) were not continuous, although variance was homogeneous 210 (Levene's test). After detecting significant effects of treatments, pairwise post-hoc 
211 Sidak multiple comparisons identified the exact time points of divergence of the 212 curves during the [T3] to [T6] follow-up period.

213 Regarding the synovial fluid analyses, the Student's t-test for paired samples (one214 sample t-test) was used to compare between experimental times within groups and 215 the unpaired t-test (two-sample t-test) for comparisons between groups. The Pearson 216 test for linear relationships between two continuous variables) was used to investigate 217 the correlations between the synovial markers, both among them and between them, 218 and the KSS or WOMAC scores at [T0] and at the end of treatment-[T2] for SF and 219 [T3] for KSS and WOMAC scores. Further statistical details are available in Ref. 8.

Ethical Considerations

223 The Institutional Review Board of the Rizzoli Orthopedic Institute reviewed all study 224 materials for ethical problems. The principles of the Declaration of Helsinki were 225 always respected. The study was registered in the ClinicalTrials.gov database of 226 privately and publicly funded clinical studies conducted worldwide 227 (ClinicalTrials.gov Identifier: NCT02417610). 


\section{RESULTS}

234 Figure 2 illustrates the overall flowchart of the 2-year study. At [T5], the patients of

235 the two groups who progressed towards T6 and the end of the study were still

236 homogeneous for age $(p=0.54)$, Kellgren-Lawrence grade $(p=0.13)$, gender $(p=0.84)$,

237 BMI $(p=1)$, weight $(p=0.86)$, and height $(p=0.67)$.

238

239

Overall flowchart of the 2-year study

\section{FIRST YEAR}

240

241

242

243

244

245

246

247

248

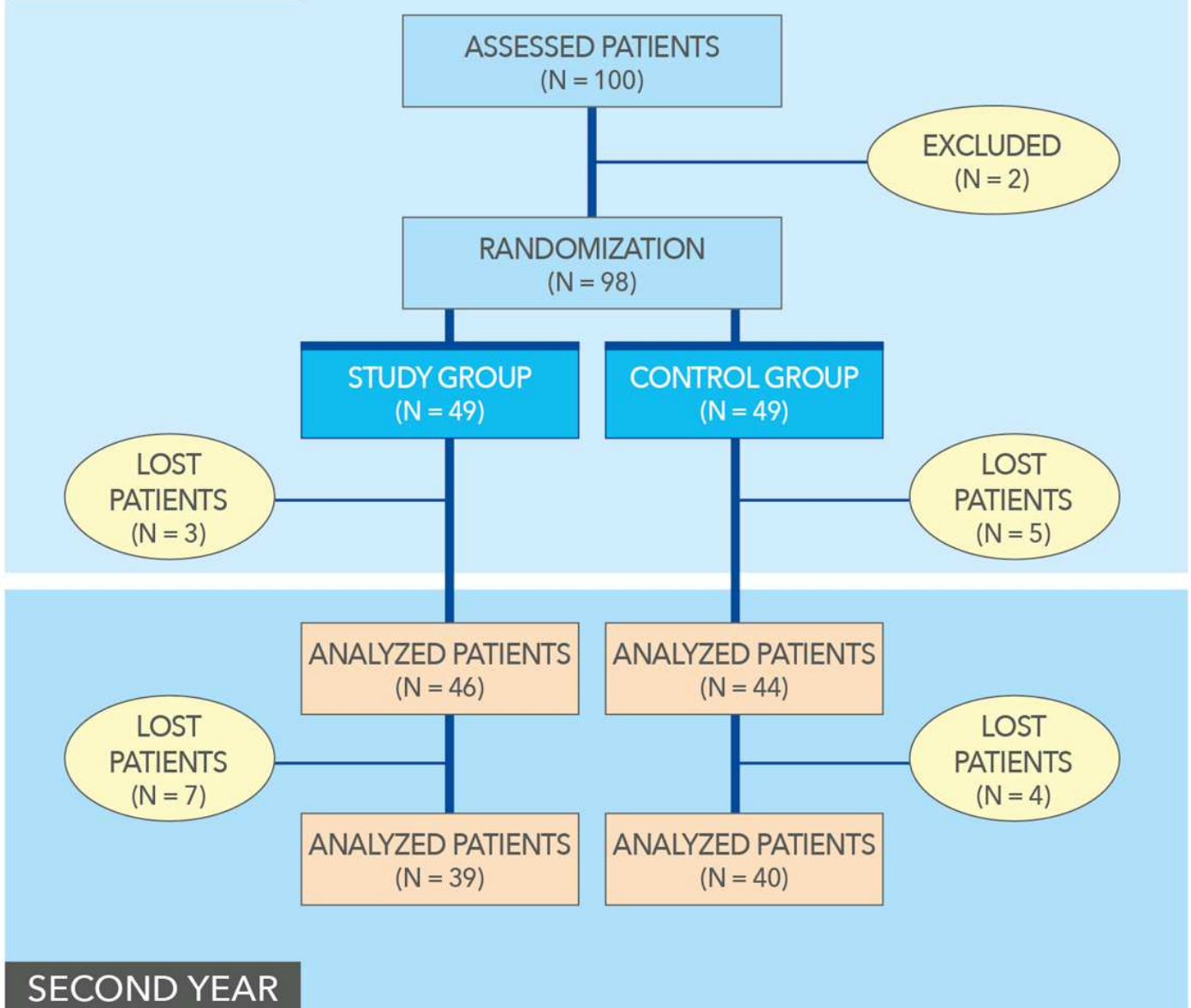

250

SECOND YEAR

251 Figure 2 Upper lighter blue area: first-year part of the study leading to the interim analysis at

252 the end of the first study year-i.e., outcomes up to [T5] or 12 months discussed in Ref. 8.

253 Lower darker blue area: second-year follow-up. 
255 As reported in the interim report, the first year of follow-up saw no infiltration-related 256 complications. ${ }^{8}$ Seventy-nine patients completed the study (39 in the PNHA group, 40

257 in the control HA group), with seven more patients lost in the PNHA group and 4 in 258 the HA group, once again due to personal reasons. As regards the primary endpoint, 259 WOMAC pain score, the pain curves were significantly different at one-way ANOVA 260 ( $p=0.029$; partial eta squared $=0.07)$; divarication of pain curves was both precocious 261 ([T3], $p=0.0006$ at Sidak test) and steady for two years $-[T 4] p=0.01,[T 5] p=0.001,[T 6]$ $262 \mathrm{p}=0.09$ (Figure 3).

263 264 Two-year WOMAC pain scores in the two treatment groups

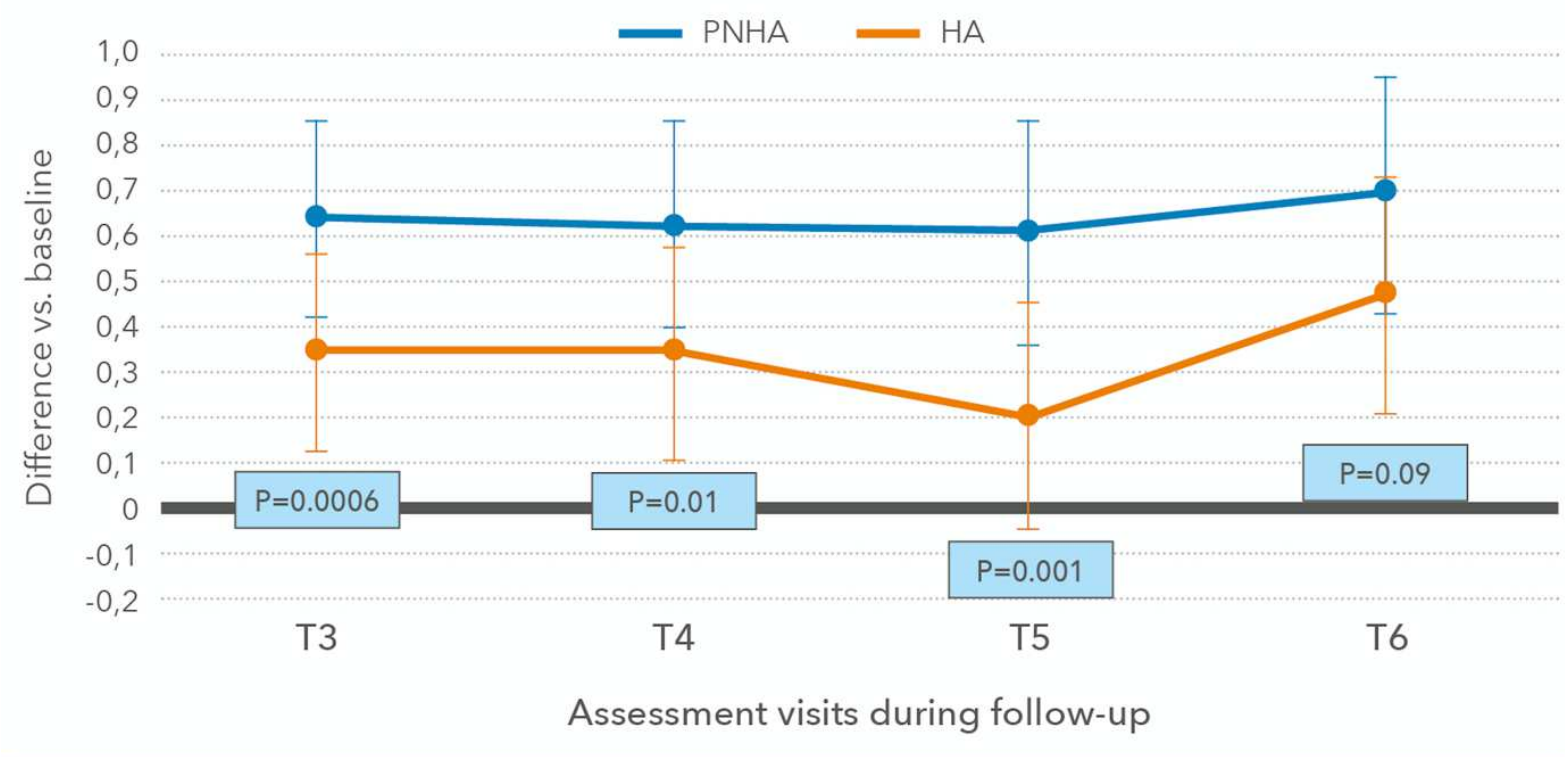

272 Figure 3 Differences in Western Ontario and McMaster Universities (WOMAC) pain scores

273 (primary endpoint; mean $\pm \mathrm{SD}$ ) vs baseline during the [T3] (2 months) to [T6] (24 months) 274 follow-up period (positive values: improvement vs baseline). 
277 The mean difference in favour of the PNHA group vs the HA control group was about

$27816 \%$. The improvement of pain showing significant differences at [T4] $(\mathrm{p}=0.029)$ and

279 [T5] $(p=0.046)$, and an almost significant difference at [T6] $(p=0.059)$. The other

280 WOMAC items did not show differences between the two groups, with the partial

281 exception of "walking on a flat surface", which was always tendentially easier for

282 patients in the PNHA group and significantly so at [T5] and [T6] (Figure 4). As a result,

283 the mean total WOMAC scores showed a tendency to improve steadily more in the

284 PNHA group than HA controls, over the whole follow-up period (Figure 5), although

285 the difference was statistically significant only at [T6] after corrections for age and 286 other parameters.

287

288

Two-year "walking on a flat surface" WOMAC subscores

289

290

291

292

293

294

295

Assessment visits during follow-up

296 Figure 4 Mean "walking on a flat surface" Western Ontario and McMaster Universities

297 (WOMAC) subscores; mean \pm SD) during the [T3] (2 months) to [T6] (24 months) follow-up

298 period (positive values: improvement vs baseline). 


\section{Two-year total WOMAC scores}

301

302

303

304

305

306

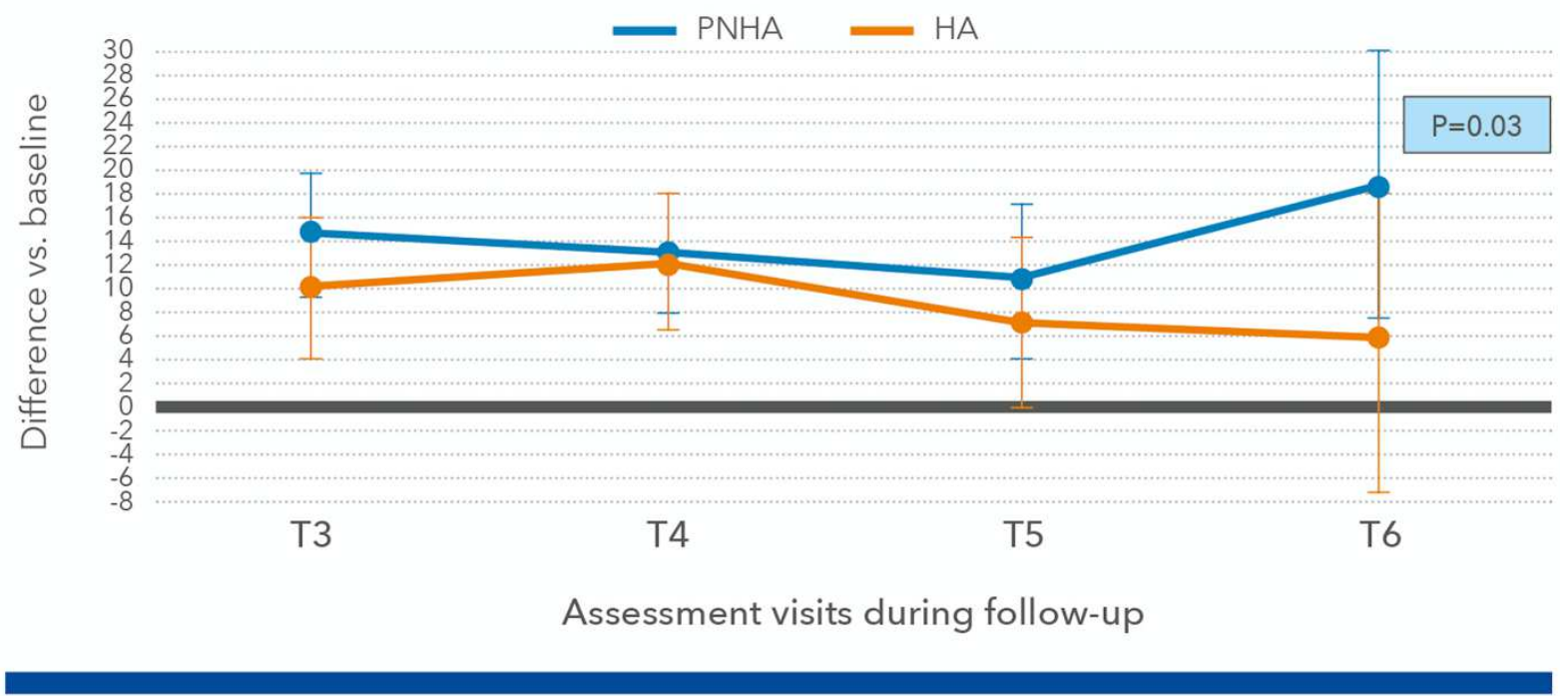

308

Figure 5 Differences in total Western Ontario and McMaster Universities (WOMAC) scores (mean \pm SD) vs baseline during the [T3] (2 months) to [T6] (24 months) follow-up period (positive values: improvement vs baseline).

313 The KSS total scores over the first year were always significantly higher in the PNHA

314 study group compared with the HA control group at all follow-up assessments

$315(p=0.02$ at [T3] and $p=0.001$ at both [T4] and [T5]). The 2-year study confirmed the

316 tendency towards a long-term pain benefit for PNHA-treated patients also at the last

317 [T6] assessment (Figure 6). 


\section{Two-year KSS scores}

322

323

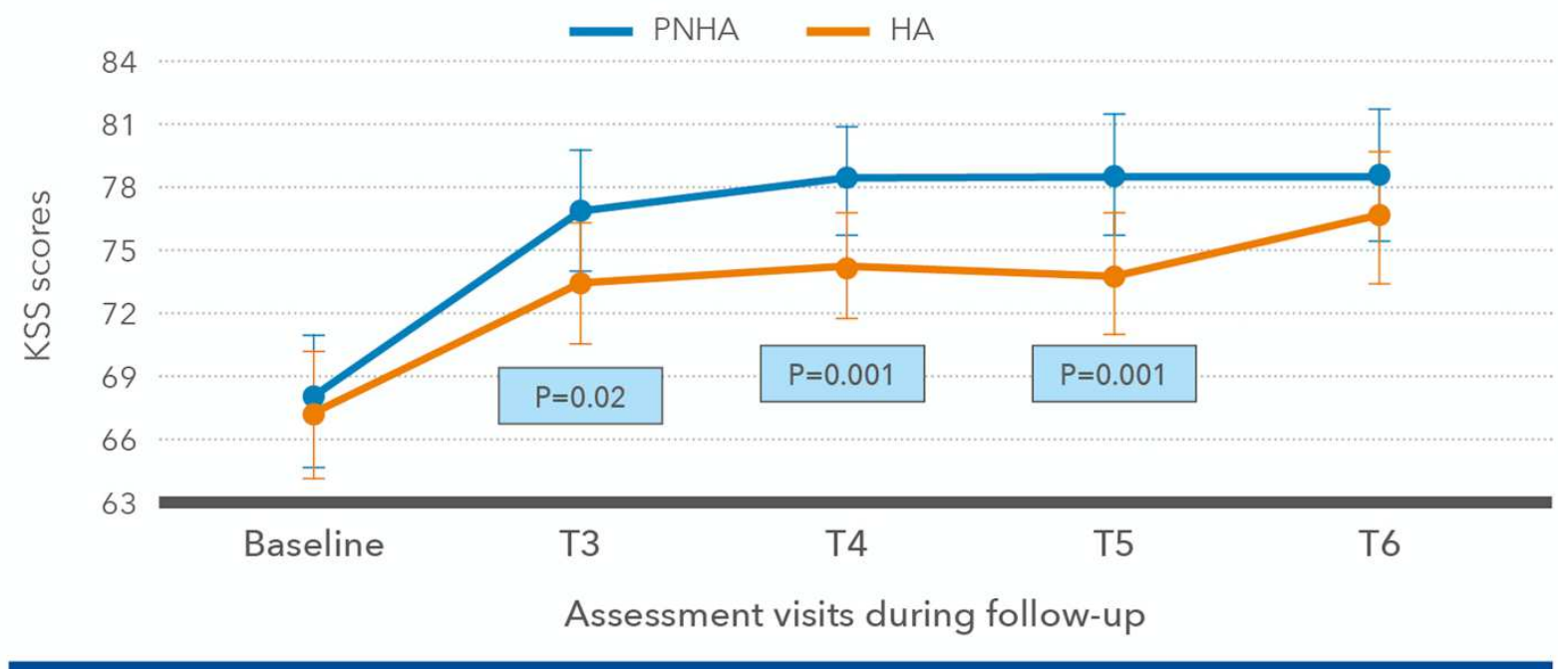

329 Figure 6 Knee Society Score (KSS) scores (mean \pm SD) during the [T3] (2 months) to [T6]

330 (24 months) follow-up period (positive values: improvement vs baseline).

332 The overall outcomes were similar for the KSS "pain" item subscore $(p<0.05$ at [T3]

333 and [T5]; [T6] p=0.059 marginally not significant), with $87 \%$ of patients of the PNHA

334 treatment group (34 out of 39) and 66\% of the HA group reporting an improvement

335 of joint pain (Figure 7).

336 


\section{Patients with improved joint pain in the two treatment groups}

344

346

347

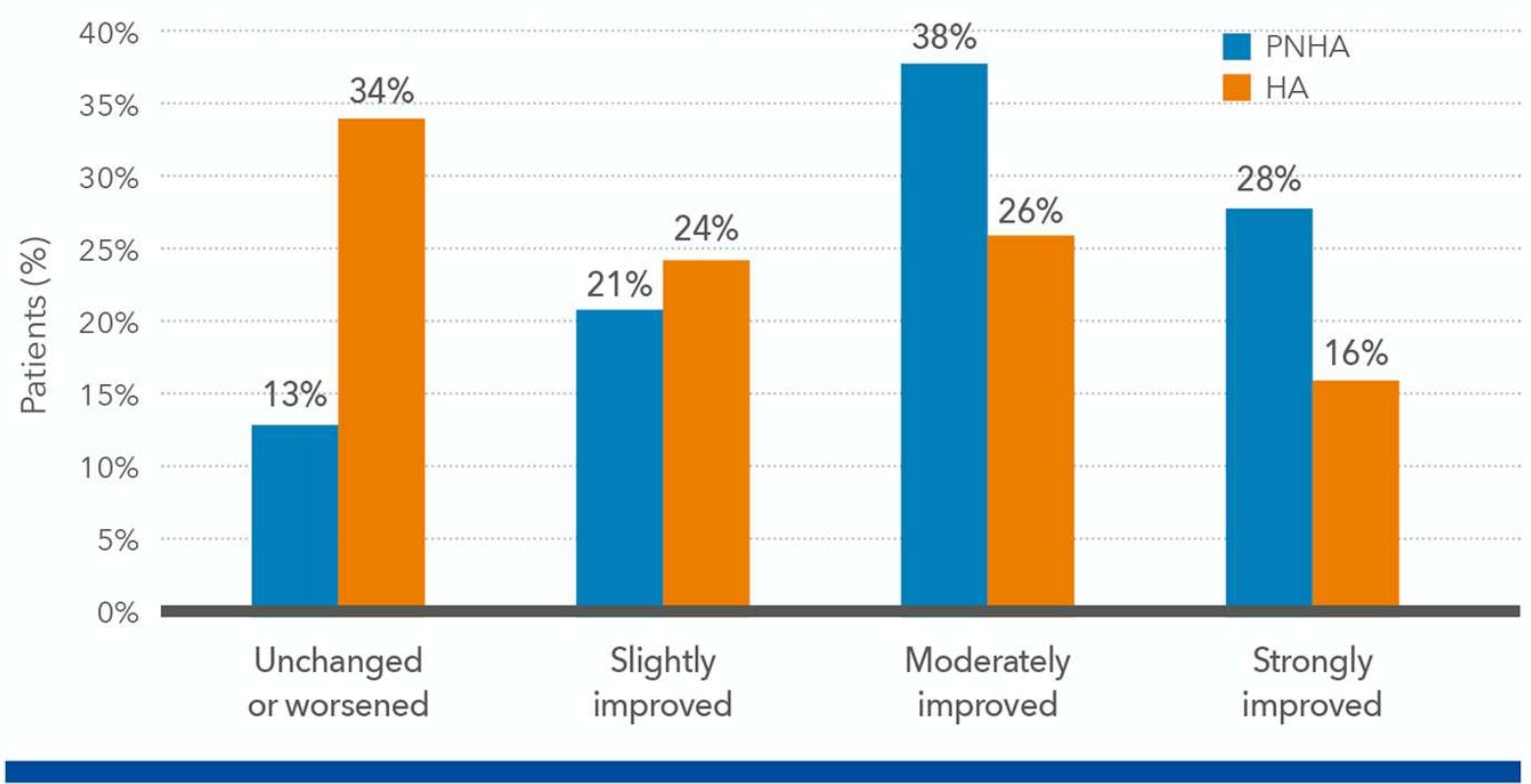

352 Figure 7 Percent of patients in the fixed combination (PNHA) and hyaluronic acid (HA)

353 treatment groups reporting improvement in Knee Society Score (KSS) pain scores during the

354 [T3] (2 months) to [T6] (24 months) follow-up period.

355

356

357 The degree of improvement in mean KSS pain scores was different in patients of the

358 PNAH treatment group and patients of the HA group as a function of joint damage

359 severity, with a more substantial decrease of pain scores in patients with more severe

360 disease (Figure 8). 


\section{KSS pain scores over the first 2 months of the study}

366

367

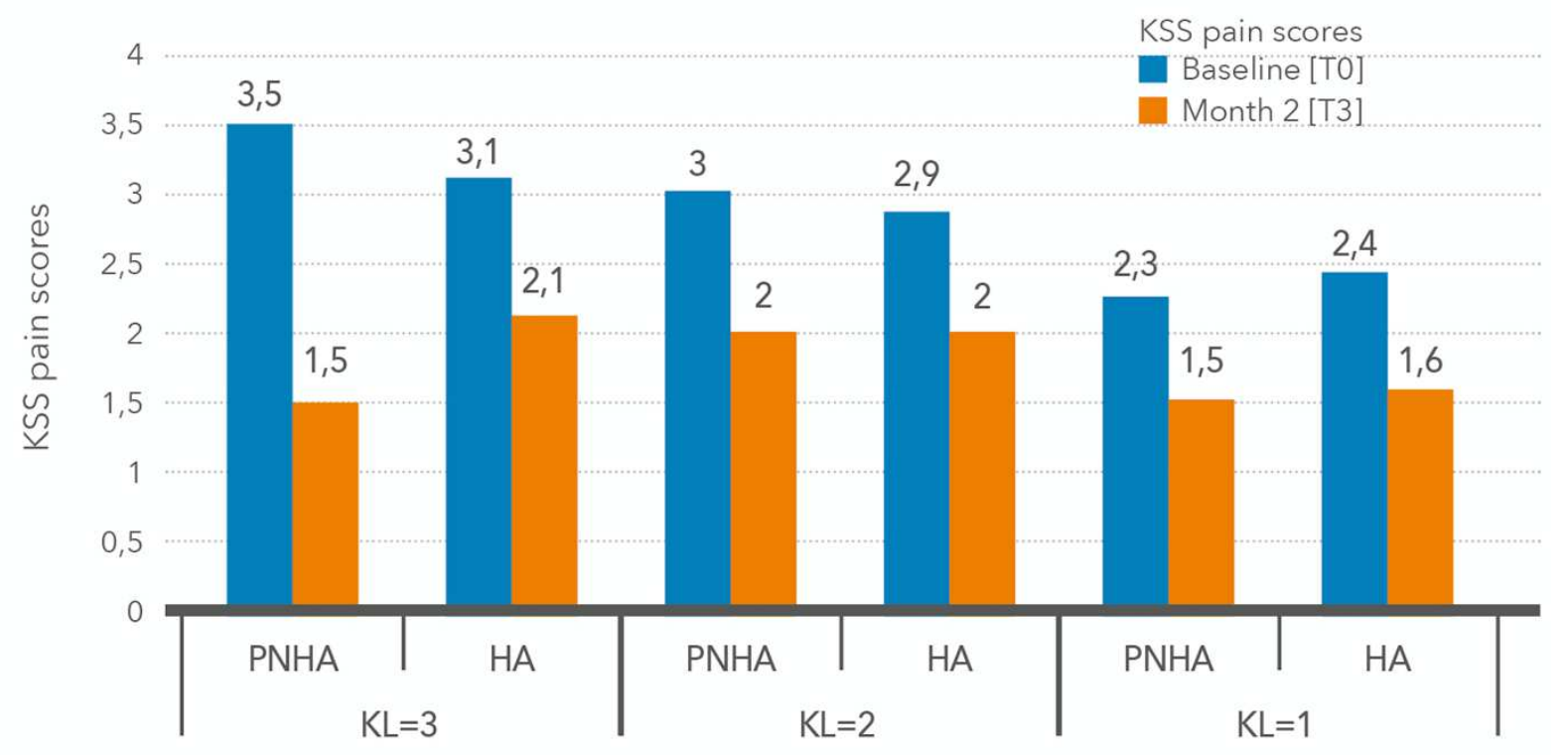

373

368

374 Figure 8 Mean Knee Society Score (KSS) pain scores at baseline and [T3] (2 months) in

375 patients of the fixed combination (PNHA) and hyaluronic acid (HA) treatment groups

376 according to baseline severity (Kellgren-Lawrence grade) of knee joint disease.

379 Mean KSS pain scores improved by 2 points both early after the end of the treatment cycle [T3] and at the end of the 2-year follow-up [T6] in PNHA-treated patients with more severe knee joint disease; conversely, KSS pain scores improved by 1 point in

382 the HA-treated patients with the same degree of disease severity (Figure 9). Mean 383 improvements were similar in patients with less severe disease; NSAIDs consumption was also similar in the two treatment groups (11 patients in both groups). 


\section{Two-year KSS pain scores vs. baseline clinical severity}

388

389

390

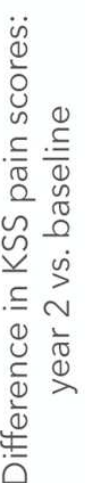

2,5

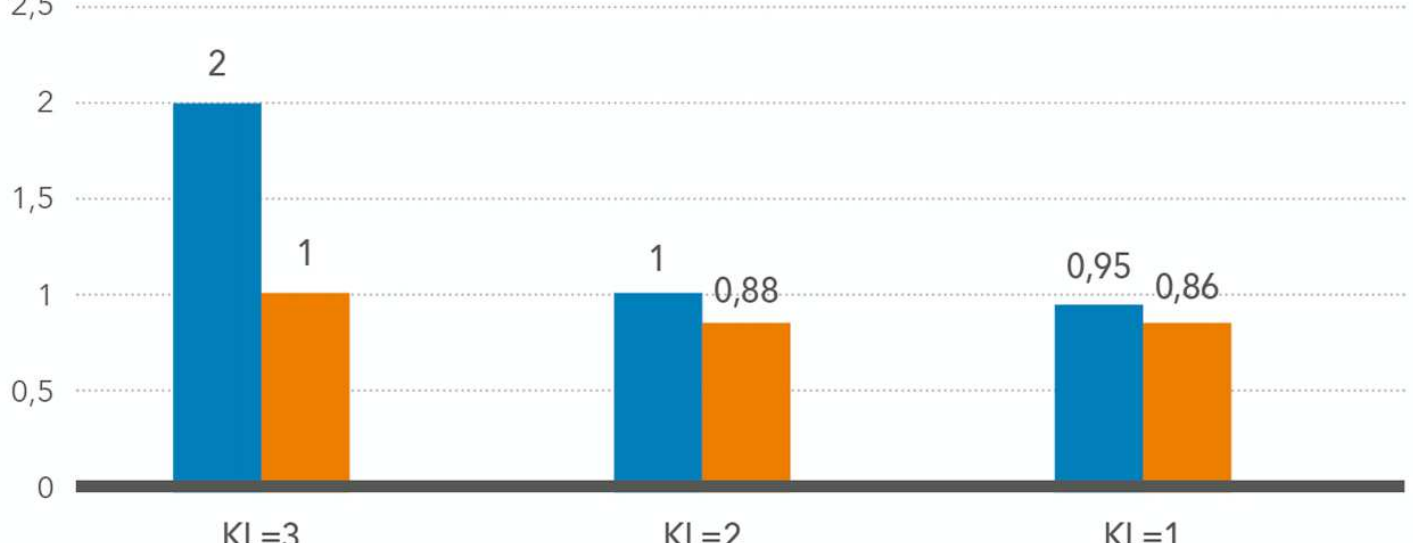

394

$\mathrm{KL}=3$

$\mathrm{KL}=2$

$\mathrm{KL}=1$

Figure 9 Improvement in mean Knee Society Score (KSS) pain scores, baseline vs [T6] (24 months) in patients of the fixed combination (PNHA) and hyaluronic acid (HA) treatment groups according to baseline severity (Kellgren-Lawrence grade) of knee joint disease.

400 The synovial fluid samples of all patients were transparent or translucent, showed a

401 well-defined clot, and were of a regular yellow or, more frequently, light yellow 402 colour. The synovial fluid clarity and density (mucin clot test) were also normal in all 403 patients. The total white cell count was always within the non-inflammatory range $(<$ 4042000 cells $/ \mathrm{mm}^{3}$ ). Synovial fluid levels of MMP1, MMP13, IL-6, TNF- $\alpha$, and PGE2 405 showed a tendency to reduction, often quite substantial compared with baseline, after 406 two months of PNHA treatment (e.g., MMP1 -49\%, MMP13 -31,2\%). Conversely, 407 MMP1, and MMP13 levels increased ( $+29,5 \%$ and $+6 \%$, respectively) and only levels of IL-6, IL-8, and PGE2 appeared reduced after treatment with HA. However, mainly 
409 due to high variability, the low number of patients eligible for synovial fluid sampling

410 and the overall low number of samples, statistical comparison of synovial fluid

411 markers did not yield significant results (data not shown). The main reason was that

412 a set of synovial fluid samples at both [T0] and [T2] was available for only eight

413 patients.

414 Neither infiltrative treatment was associated with short-term complications or long-

415 term side effects of any clinical significance.

\section{DISCUSSION}

419 The final two-year outcomes of this randomised, double-blind study confirm the 420 preliminary outcomes of the previous 1-year interim report-the intra-articular co-

421 administration of a fixed combination of PN-HPT ${ }^{\mathrm{TM}}$ and HA is associated with 422 significant benefits for the knee joint pain, the primary study endpoint, and functional 423 disabilities compared with HA alone. ${ }^{8}$ The final two-year outcomes of the study also 424 support the rationale that inspired the development of the fixed PNHA combination425 synergy between PN-HPT ${ }^{\mathrm{TM}}$ and HA is likely in OA based on the complementary 426 properties of the two viscoelastic agents. ${ }^{8}$

427 Highly purified, natural-origin PN-HPT ${ }^{\mathrm{TM}}$ - linear chains of polynucleotides from 428 trout gonads - release nucleosides, nucleotides, and nitrogen bases by enzymatic 429 cleavage in the synovial space and have shown long-term moisturising, and 430 viscoelastic properties in clinical studies in knee OA.-6 ${ }^{4 N-H_{P T}{ }^{\mathrm{TM}}}$ combine these 
431 properties with a robust trophic activity on mesenchymal tissues and cells and 432 protection of cartilage. ${ }^{7,12}$ The biostimulating efficacy of PN-HPT ${ }^{\mathrm{TM}}$ appears stronger 433 than HA, which supports the "PN-HPT" plus HA" synergy concept that inspired the 434 2-year study herein discussed. ${ }^{8} \mathrm{PN} \mathrm{HPT}{ }^{\mathrm{TM}}$ also seem to have more substantial pain435 reduction properties than $\mathrm{HA}$ in patients with knee OA. ${ }^{4}$

436 The more rapid reduction of WOMAC pain scores, the primary endpoint of the study, 437 in PNHA-treated patients compared with the HA group is likely to mirror the 438 synergic short-term viscoelastic contribution of $\mathrm{PN}-\mathrm{HPT}^{\mathrm{TM}}$ to the investigated fixed 439 formulation. Such synergy also likely explains the steady long-term reduction of knee 440 pain, substantial at [T4] and [T5] (months 6 and 12) compared with HA-treated 441 patients, but extending over the whole two-year study period. Without that synergy 442 in the HA treatment group, pain significantly decreased only at the second and fourth 443 months of follow-up ([T3] and [T4]), but not after 12 ([T5]) and 24 months ([T6]). Of 444 course, the use in controls of a low-molecular-weight HA, which may have low 445 elastoviscosity and require frequent infiltrations, might have acted as a confounding 446 factor. ${ }^{13}$ The benefits for the WOMAC item "Walking on a flat surface" developed 447 somewhat more slowly in PNHA-treated patient, with still no differences between 448 PNHA and HA at [T3] and [T4], but statistically significant ones at both [T5] and [T6]. 449 PN-HPT ${ }^{\mathrm{TM}}$ improved knee OA symptoms more effectively, and possibly earlier than 450 HA in patients with high-grade chondropathy, thus confirming previous 451 observations.5,6 More specifically, the PNHA treatment group experienced more 452 substantial two-year reductions of both KSS and especially WOMAC mean pain 
453 subscores than the HA treatment group. Pain benefits, already manifest in patients

454 with the least severe disease (KL grade 1), increased progressively with disease 455 severity, from KL grade 1 up to KL grade 3. PN-HPT ${ }^{\mathrm{TM}}$ strongly inhibits the migration

456 of inflammatory cells and the local expression of inflammatory markers, and this

457 might be the basis of such reasonable pain control despite advanced joint damage. ${ }^{14,15}$

458 A retrospective stratification of OA severity supports the former observation about

459 the comparative pain benefits progressively increasing in grade-1, grade- 2 and grade-

4603 OA patients. The observation is also limited to pain, meaning caution is warranted.

461 However, compounding this clinical retrospective observation with the PN-HPT

462 characteristics described in the literature may help conceive a tentative decisional

463 algorithm to help choose between PNHA or HA in daily clinical practice (Figure 10),

464 with the PNHA doses

465 in the range 2 to $4 \mathrm{~mL}$.

466

467

468 Figure 10 Does an

469 ideal knee OA patient

470 for either PNHA or HA

471 exist? A tentative

472 decisional algorithm.

\section{Choosing between PNHA and HA}

Knee OA patients

KL grade 1-3; grade 4 waiting for TKA

\section{YES}

Advanced disease?

e.g., $K L$ degree $\geq 2^{8}$

\section{YES}

Signs of inflammation? ${ }^{8}$

- Local

(e.g., rubor and calor)

- Systemic

(e.g., elevated ESR or CRP)

\section{YES}

Fastidious or resistant knee pain or severely compromised joint function? ${ }^{8}$

Either intra-articular HA

or PNHA to leverage

polynucleotide potential on mild symptoms
Either intra-articular $\mathrm{HA}$

or PNHA to leverage trophic potential on chondrocytes

Either intra-articular $\mathrm{HA}$ or PNHA to leverage soothing potential on mild inflammation

\section{YES}

Intra-articular PNHA 
474 Regarding the still debated association between pain and synovial fluid inflammation,

475 the one-year interim report discussed assays' rationale. ${ }^{8}$ IL1- $\Omega$, TNF- $\alpha$ and IL-6 are the 476 proinflammatory cytokines most frequently associated with OA severity, while 477 MMP13 is a primary culprit of the severe damages to joint cartilages. ${ }^{16-18}$ The analysis 478 indeed found an inverse correlation between the total KSS score and IL-6 and a trend 479 towards reduced MMP1 and MMP13 synovial levels in the PNHA treatment group. 480 However, no statistical correlation existed with clinical parameters, possibly due to 481 the low number of synovial fluid samples and the short treatment period. As stated 482 in the previous interim report, detecting clinically relevant differences in synovial 483 fluid inflammatory markers might have required more follow-up time after the 484 treatment cycle and more synovial fluid samples. ${ }^{8}$

485 As a final consideration, the authors acknowledge some weak points of their study: 486 for instance, a three-edged, parallel-group study - placebo, PN-HPT ${ }^{\mathrm{TM}}$, PNHA 487 would have been more discriminating and informative. The study's primary purpose 488 was to identify a role, if any, and possibly a therapeutic niche for PNHA in the current 489 HA-dominated landscape, leading to the two-group design. The authors feel the study 490 fulfilled this limited goal; other considerations, including pharmacoeconomics, will 491 have to wait for future studies. The low mean clinical severity of enrolled patients (2 $492 \pm 0.7$ for all patients, $1.9 \pm 0.6$ for the PNHA group) is possibly another weak point. 493 Incorporating more grade-3 patient would have been likely more discriminating in a 494 study of such ambition. 
495 A third point liable to criticism: why falling back to traditional radiology instead of 496 evaluating cartilage trophism with a rapid magnetic resonance imaging technique like 497 3T MRI? The reason was simple: even in an excellence centre, the risk that MRI 498 resources were overburdened was steadily substantial over the study years.

499 Summarising, as shown by the two-year evolution of the primary endpoint, the 500 WOMAC pain score, the study demonstrated a steady, long-term improvement of 501 OA-related knee pain in PNHA-treated patients. The pain benefit vs HA was 502 significant at all assessment times and greater in patients with a high KL degree of 503 basal OA severity. Conversely, WOMAC pain control was somewhat unsteady in 504 many patients of the HA treatment group, worsening after six months and one year 505 of follow-up, and, at least tendentially, even after two years. Although some 506 secondary endpoints did not show significant differences, KSS pain control was more 507 rapid, already after two months after the end of the treatment cycle, in PNHA-treated 508 patients.

\section{CONCLUSIONS}

512 The two-year, double-blind study outcomes confirmed natural-origin, highly purified 513 polynucleotides (PN-HPT ${ }^{\mathrm{TM}}$ ) as agents with long-acting viscosupplementation 514 properties and persistent pro-trophic and protective activity on chondrocytes, and a 515 valuable complement to HA for the relief of pain and functional symptoms in knee 516 OA. The suggested PNHA therapeutic range is 2 to $4 \mathrm{~mL}$, but even the lowest dose 
517 used in the trial $(2 \mathrm{~mL})$ led to the observed favourable results. The vigorous PN-HPT 518 trophic activity on all connective tissues, including joint cartilage, might be especially 519 of value as the basis of the likely in-vivo synergy between the two viscoelastic agents.

\section{LIST OF ABBREVIATIONS}

524 3T MRI 3-Tesla Magnetic Resonance Imaging

525 BMI Body Mass Index

526 CRP C-Reactive Protein

$527 \quad \mathrm{kDa} \quad$ kilodalton

$528 \quad \mathrm{E}_{2} \quad$ Prostaglandin E2

529 ESR Erythrocyte Sedimentation Rate

530 HA Hyaluronic Acid

531 KL Kellgren-Lawrence grade

532 KSS Knee Society Score

533 IL-1ß Interleukin $1 ß$

$534 \quad$ IL-6 Interleukin 6

535 IL-8 Interleukin 8 (chemokine)

536 MMP1 Matrix Metalloproteinase-1

537 MMP13 Matrix Metalloproteinase-13

538 NSAID Non-Steroidal Anti-Inflammatory Drugs

539 OA Osteoarthritis

540 OARSI Osteoarthritis Guidelines Development Group 
541 PNs Polynucleotides

542 PNHA PNs and HA fixed combination

543 PN-HPT ${ }^{\mathrm{Tm}}$ Polynucleotides "Highly Purified Technology"

544 TIMP Tissue Inhibitor of MMP1

545 TKA Total Knee Arthroplasty

546 TNF- $\alpha \quad$ Tumor Necrosis Factor- $\alpha$

547 WOMAC Western Ontario and McMaster Universities

548

549

550

551

552

553 DECLARATIONS

554 Ethics approval and consent to participate

555 The Institutional Review Board of the Rizzoli Orthopedic Institute reviewed all study

556 materials such as study protocol, informed consent forms, electronic clinical report

557 form, CVs of authors, Etc. for ethical problems. All relevant documents (IRB approval

558 certificate and approved study materials) are available from Dr Dante Dallari or the

559 corresponding author, Martina Rocchi, MD, on request. The principles of the

560 Declaration of Helsinki were always respected. The study was registered in the

561 ClinicalTrials.gov database of privately and publicly funded clinical studies

562 conducted worldwide (ClinicalTrials.gov Identifier: NCT02417610). 


\section{Consent for publication}

565 The manuscript contains no individual patient's data in any form - all authors consent

566 to the manuscript's publication.

567

\section{Availability of data and material}

569 The datasets generated and analysed during the current study, not publicly available, 570 are currently archived according to current regulations (with full personal details of

571 all participating subjects) at the Rizzoli Orthopedic Institute, Bologna, Italy. All the

572 datasets are available (after conversion in anonymous form) from the corresponding 573 author on reasonable request.

574

\section{Competing interests}

576 The authors declare that they have no competing or conflicts of interest.

\section{$578 \quad$ Funding}

579 The study was spontaneous and touched off by scientific curiosity arising from 580 available medical literature. The corporate sponsor, Mastelli S.r.l., Sanremo, Italy, 581 provided the unidentifiable code-numbered study samples and financed all the third582 part activities associated with the study - design and discussion of the study protocol, 583 electronic clinical report forms and documents provided to the Ethical Committee 584 (available on request), as well as of all other study materials, e.g., for randomisation 
585 procedures, monitoring, and reporting. The only other funding will be the sponsor's

586 financing the article processing charges by BMC Musculoskeletal Disorders or the

587 journal that will accept the manuscript (see also "Acknowledgements" subsection).

588

589 Authors' contributions

590 All authors sought and got informed consents by all their knee osteoarthritis patients

591 enrolled in the study. After explaining the benefits and risks they could reasonably

592 expect from intra-articular infiltrations of the two study formulations, they received

593 informed consents from all candidate patients. All authors also personally carried out

594 all double-blind procedures, including baseline and follow-up WOMAC and KSS

595 scoring interviews, at both study sites, always under Dr Dallari's supervision. Paola

596 Torricelli, MSc, also blinded to individual treatments, was responsible for laboratory

597 assays.

598 Dr Dante Dallari is personally accountable for the clinical and editorial work's 599 accuracy and integrity, leading to the manuscript's submission to BMC Musculoskeletal 600 Disorders, including the comments on outcomes expressed in his manuscript.

601

602 Acknowledgements

603 Mastelli S.r.l., Sanremo, Italy, is the patent holder and producer of POLIART ${ }^{\circledR}$, 604 proprietary fixed co-formulation of polynucleotides and hyaluronic acid for intra605 articular infiltration, and IALART ${ }^{\circledR}$, a proprietary formulation of low-molecular606 weight hyaluronic acid for intra-articular infiltration. Both formulations were used 
607 and compared in the two-year study. The authors wish to acknowledge the 608 contribution of Mastelli S.r.l. for providing all materials for performing the double609 blind study together with some minor financial support to help with the publication 610 costs of the final report of their two-year study (see also "Funding" section).

611 The authors also wish to thank Dr Mauro Raichi, clinical pharmacologist, for support 612 in medical writing.

613

614

615

616

617

\section{REFERENCES}

619 1. McAlindon TE, Bannuru RR, Sullivan MC, Arden NK, Berenbaum F, Bierma-Zeinstra

620 SM, et al. OARSI guidelines for the non-surgical management of knee osteoarthritis.

621 Osteoarthritis Cartilage. 2014;22:363-88.

622 2. Henrotin Y, Raman R, Richette P, Bard H, Jerosch J, Conrozier T, et al. Consensus

623 statement on viscosupplementation with hyaluronic acid for the management of

624 osteoarthritis. Semin Arthritis Rheum. 2015;45:140-9.

625 3. Bannuru RR, Osani MC, Vaysbrot EE, Arden NK, Bennell K, Bierma-Zeinstra SMA, et al.

626 OARSI guidelines for the non-surgical management of knee, hip, and polyarticular

627 osteoarthritis. Osteoarthritis Cartilage. 2019;27:1578-89. 
4. Vanelli R, Costa P, Rossi SMP, Benazzo F. Efficacy of intra-articular polynucleotides in the treatment of knee osteoarthritis: a randomised, double-blind clinical trial. Knee Surg Sports Traumatol Arthrosc. 2010;18:901-7.

5. Saggini R, Di Stefano A, Cavezza T, Saladino G, Bellomo RG. Intrarticular treatment of osteoarthropathy knee with polynucleotides: a pilot study with medium-term follow-up. J Biol Regul Homeost Agents. 2013;27:543-9.

6. Giarratana LS, Marelli BM, Crapanzano C, De Martinis SE, Gala L, Ferraro M, et al. A randomized, double-blind clinical trial on the treatment of knee osteoarthritis: the efficacy of polynucleotides compared to standard hyaluronan viscosupplementation. Knee. 2014;21:661-8.

7. Guizzardi S, Uggeri J, Belletti S, Cattarini G. Hyaluronate increases polynucleotides effect on human cultured fibroblasts. J Cosmet Dermatol Sci Appl. 2013;3:124-8.

8. Dallari D, Sabbioni G, Del Piccolo N, Carubbi C, Veronesi F, Torricelli P, Fini M. Efficacy of intra-articular polynucleotides associated with hyaluronic acid versus hyaluronic acid alone in the treatment of knee osteoarthritis: a randomised, double-blind, controlled clinical trial. Clin J Sport Med. 2020;30:1-7.

9. Kohn MD, Sassoon AA, Fernando ND. Classifications in Brief: Kellgren-Lawrence Classification of Osteoarthritis. Clin Orthop Relat Res. 2016;474:1886-93.

10. Insall JN, Dorr LD, Scott RD, Scott WN. Rationale of the Knee Society clinical rating system. Clin Orthop Relat Res. 1989;248:13-4.

11. Bellamy N, Buchanan WW, Goldsmith CH, Campbell J, Stitt LW. Validation study of WOMAC: a health status instrument for measuring clinically important patient relevant outcomes to antirheumatic drug therapy in patients with osteoarthritis of the hip or knee. J Rheumatol. 1988;15:1833-40. 
652 12. Gennero L, Denysenko T, Calisti GF, Vercelli A, Vercelli CM, Amedeo S, et al. Protective

653 effects of polydeoxyribonucleotides on cartilage degradation in experimental cultures.

$654 \quad$ Cell Biochemistry and Function. 2013;31:214-27.

655 13. Fakhari A, Berkland C. Applications and merging trends of hyaluronic acid tissue

656 engineering, as a dermal filler and in osteoarthritis treatment. Acta Biomater.

$657 \quad 2013 ; 9: 7081-92$.

658 14. Bitto A, Polito F, Irrera N, D’ Ascola A, Avenoso A, Nastasi G, et al.

659 Polydeoxyribonucleotide reduces cytokine production and the severity of collagen-

660 induced arthritis by stimulation of adenosine $\mathrm{A}(2 \mathrm{~A})$ receptor. Arthritis Rheum.

$661 \quad 2011 ; 63: 3364-71$.

662 15. Chung KI, Kim HK, Kim WS, Bae TH. The effects of polydeoxyribonucleotides on the 663 survival of random pattern skin flaps in rats. Arch Plast Surg. 2013;40:181-6.

664 16. Daghestani HN, Kraus VB. Inflammatory biomarkers in osteoarthritis. Osteoarthritis 665 Cartilage. 2015;23:1890-6.

666 17. Heidari B, Hajian-Tilaki K, Babaei M. Determinants of pain in patients with symptomatic 667 knee osteoarthritis. Caspian J Intern Med. 2016;7:153-61.

668 18. Akhtar N, Khan NM, Ashruf OS, Haqqi TM. Inhibition of cartilage degradation and 669 suppression of PGE2 and MMPs expression by pomegranate fruit extract in a model of 670 posttraumatic osteoarthritis. Nutrition. 2017;33:1-13. 


\section{Treatment schedule and assessments over 2 years}

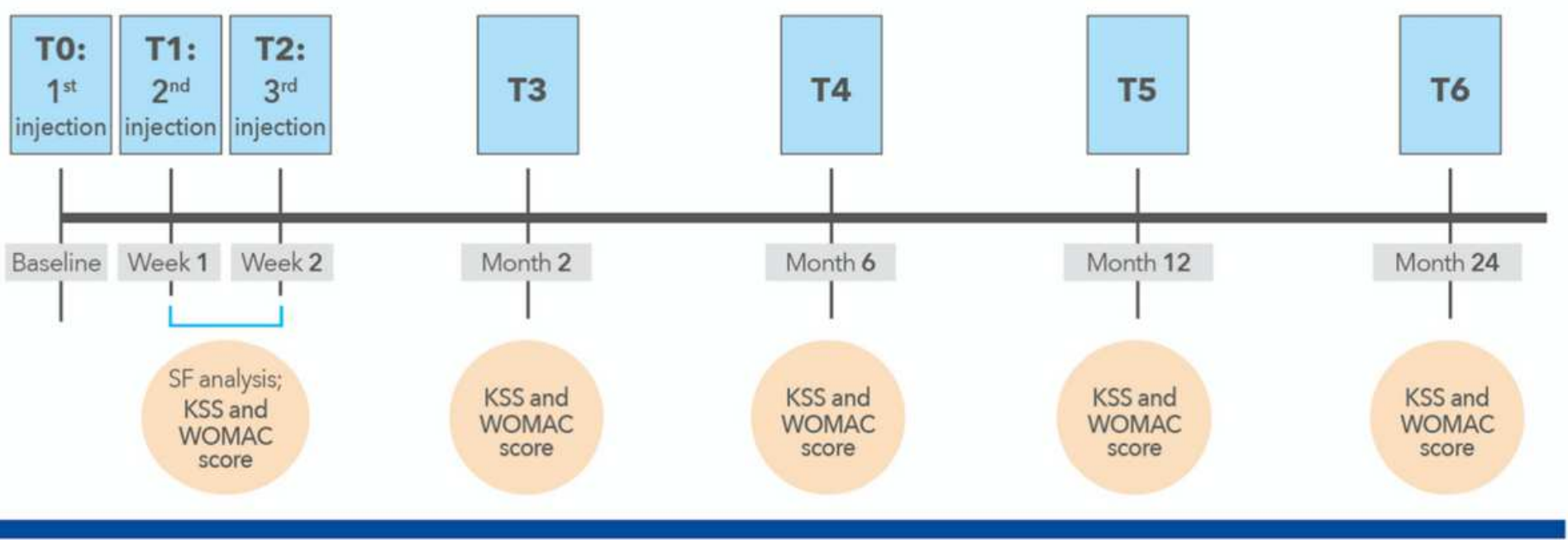

\section{Figure 1}

[T0] to [T2]: timing of the three double-blind PNHA and HA intra-articular injections and assessments planned over the first two study weeks (synovial fluid analysis and first KSS and WOMAC evaluation); [T3] to [T6]: timing of the KSS and WOMAC evaluations planned over the residual 2-year study period. 


\section{Overall flowchart of the 2-year study}

\section{FIRST YEAR}

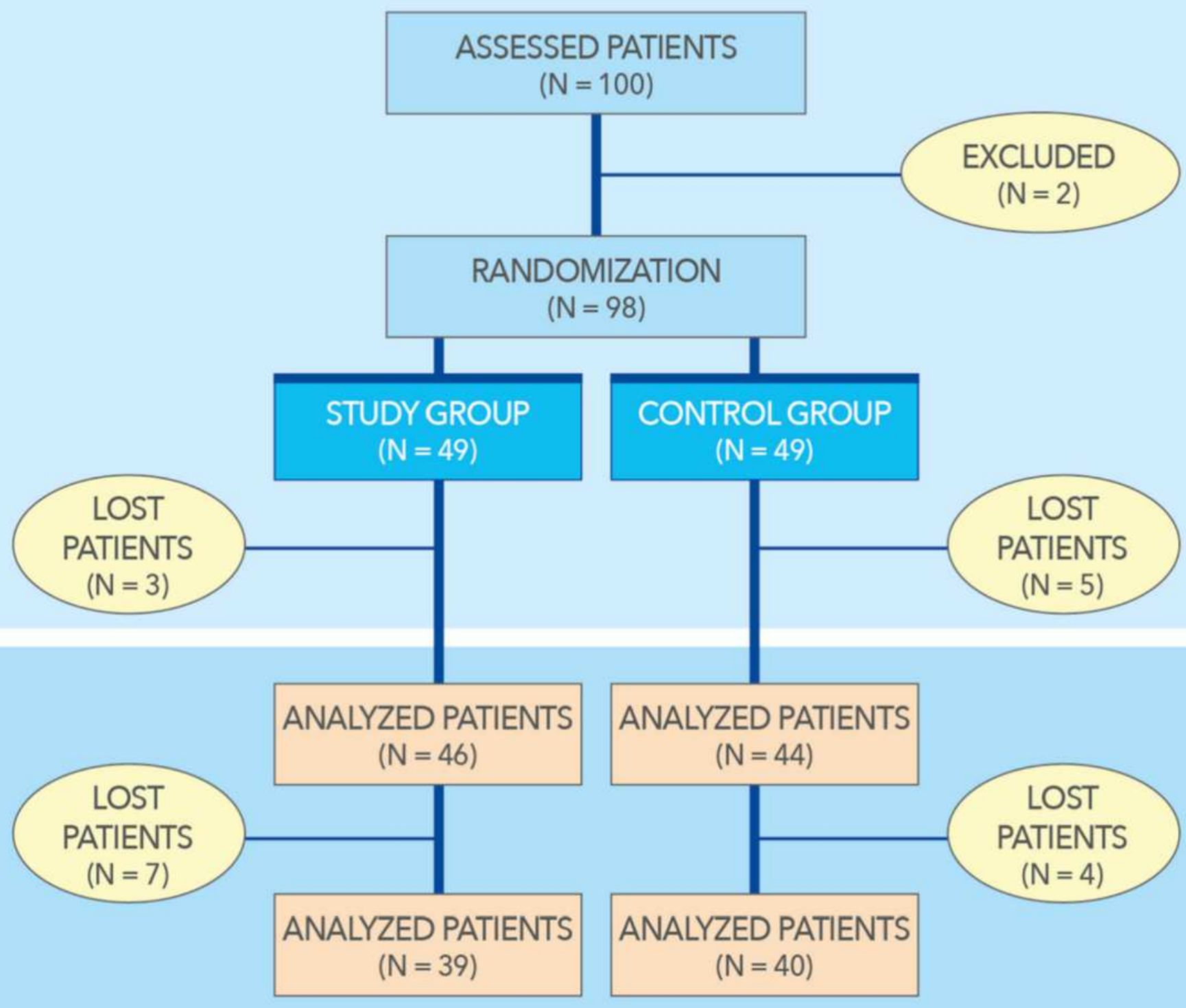

\section{SECOND YEAR}

Figure 2

Upper lighter blue area: first-year part of the study leading to the interim analysis at the end of the first study year-i.e., outcomes up to [T5] or 12 months discussed in Ref. 8. Lower darker blue area: secondyear follow-up. 


\section{Two-year WOMAC pain scores in the two treatment groups}

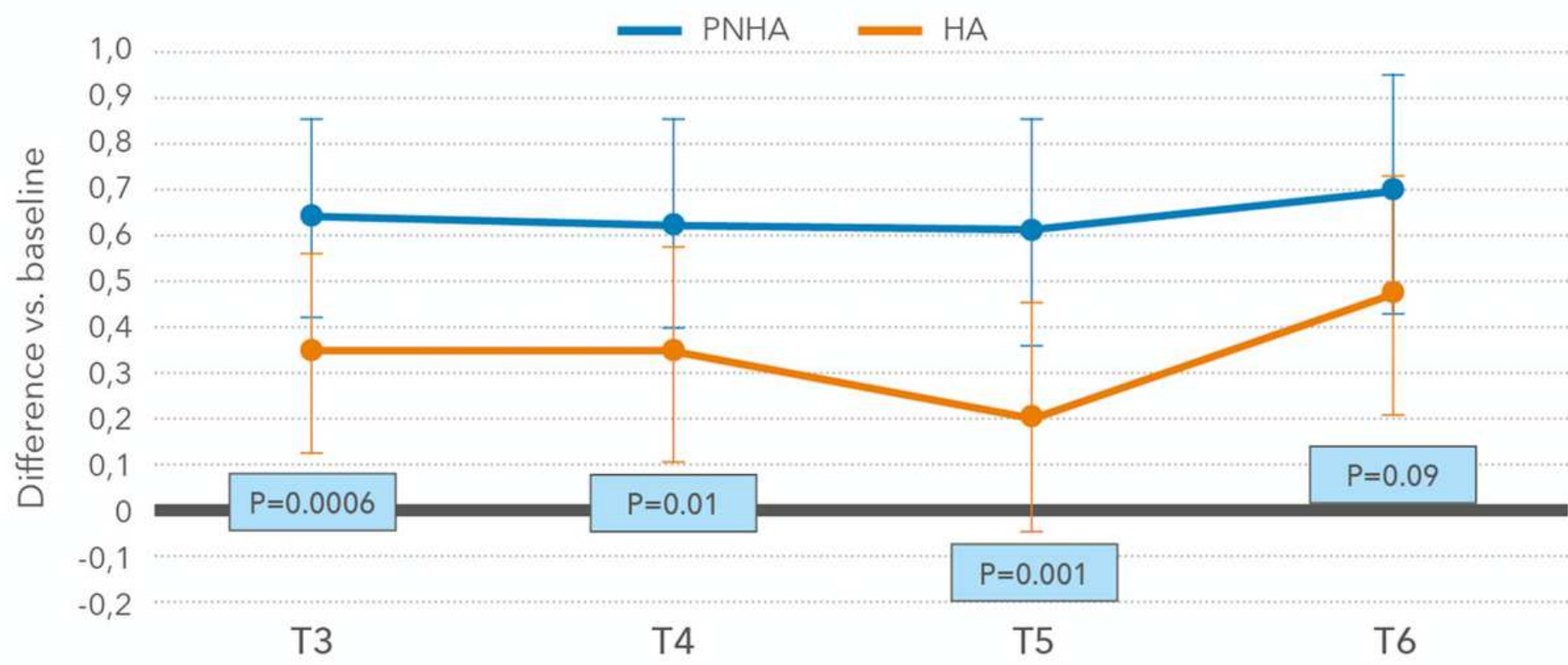

Assessment visits during follow-up

\section{Figure 3}

Differences in Western Ontario and McMaster Universities (WOMAC) pain scores (primary endpoint; mean \pm SD) vs baseline during the [T3] (2 months) to [T6] (24 months) follow-up period (positive values: improvement vs baseline). 


\section{Two-year "walking on a flat surface" WOMAC subscores}

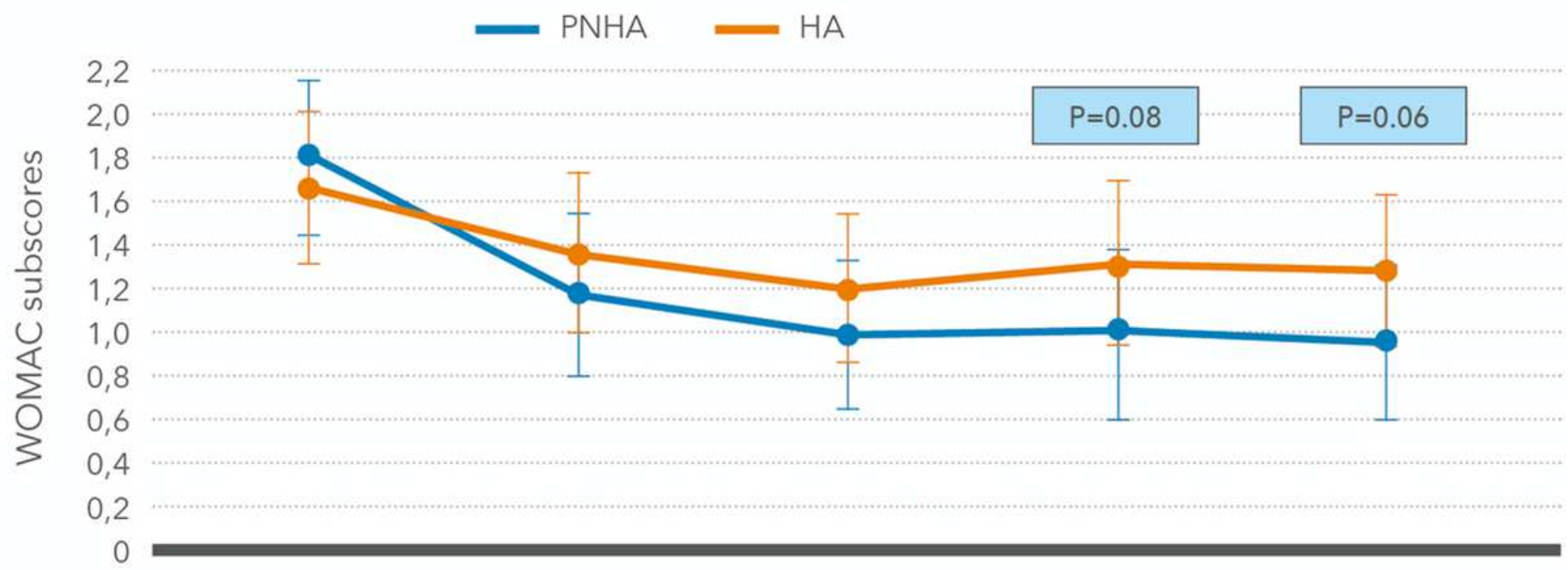

Assessment visits during follow-up

\section{Figure 4}

Mean "walking on a flat surface" Western Ontario and McMaster Universities (WOMAC) subscores; mean \pm SD) during the [T3] (2 months) to [T6] (24 months) follow-up period (positive values: improvement vs baseline). 


\section{Two-year total WOMAC scores}

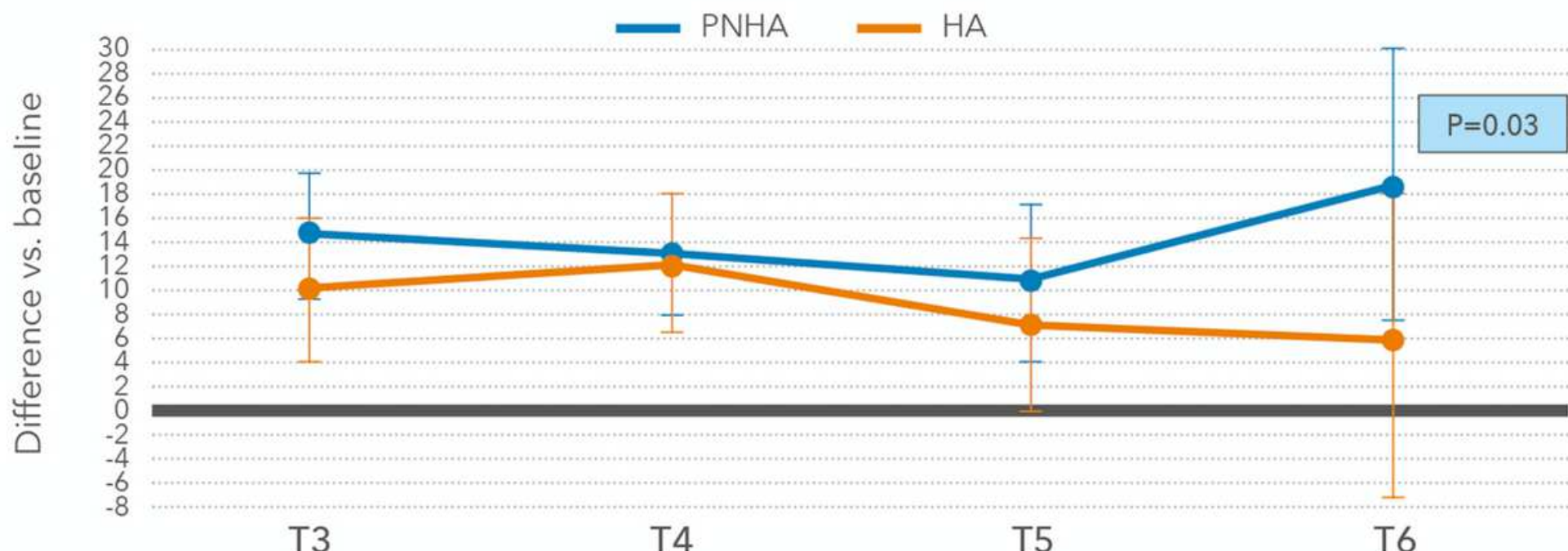

Assessment visits during follow-up

\section{Figure 5}

Differences in total Western Ontario and McMaster Universities (WOMAC) scores (mean \pm SD) vs baseline during the [T3] (2 months) to [T6] (24 months) follow-up period (positive values: improvement vs baseline). 


\section{Two-year KSS scores}

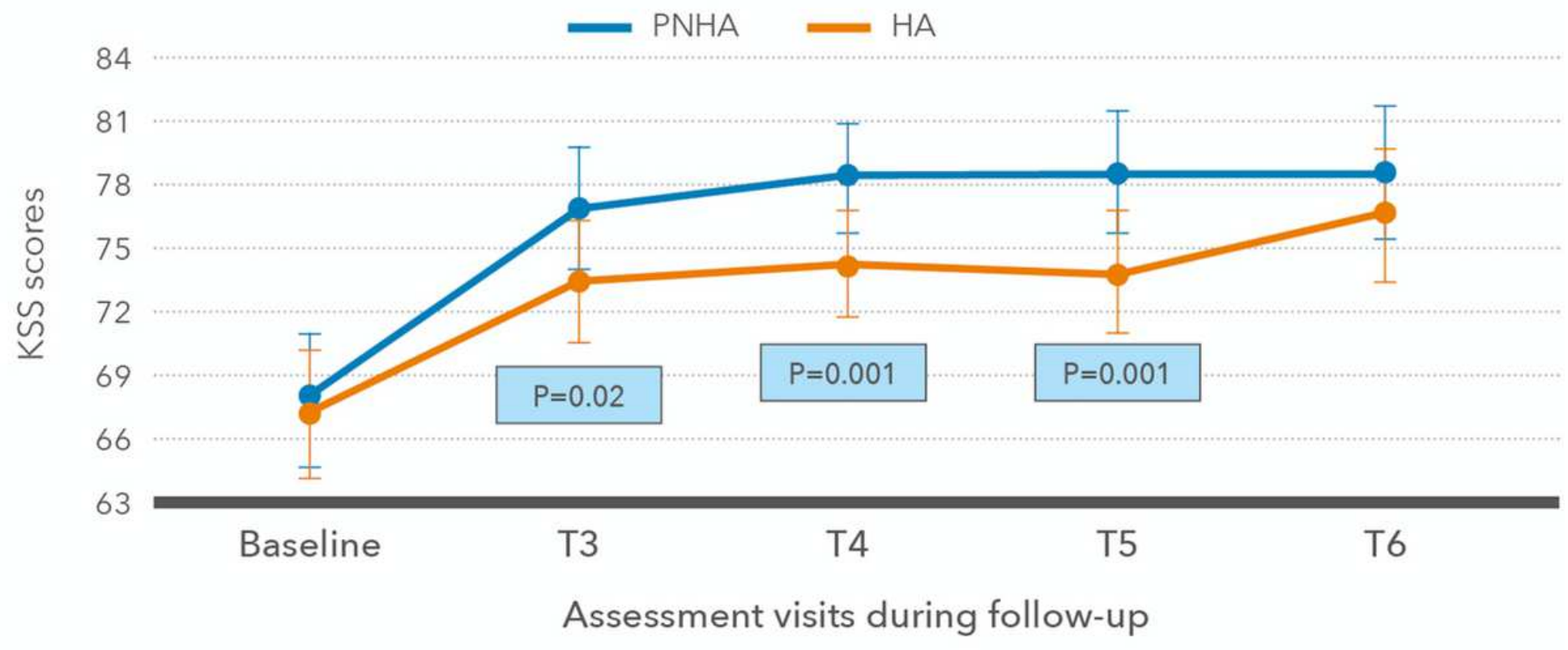

Figure 6

Knee Society Score (KSS) scores (mean \pm SD) during the [T3] (2 months) to [T6] (24 months) follow-up period (positive values: improvement vs baseline). 


\section{Patients with improved joint pain in the two treatment groups}

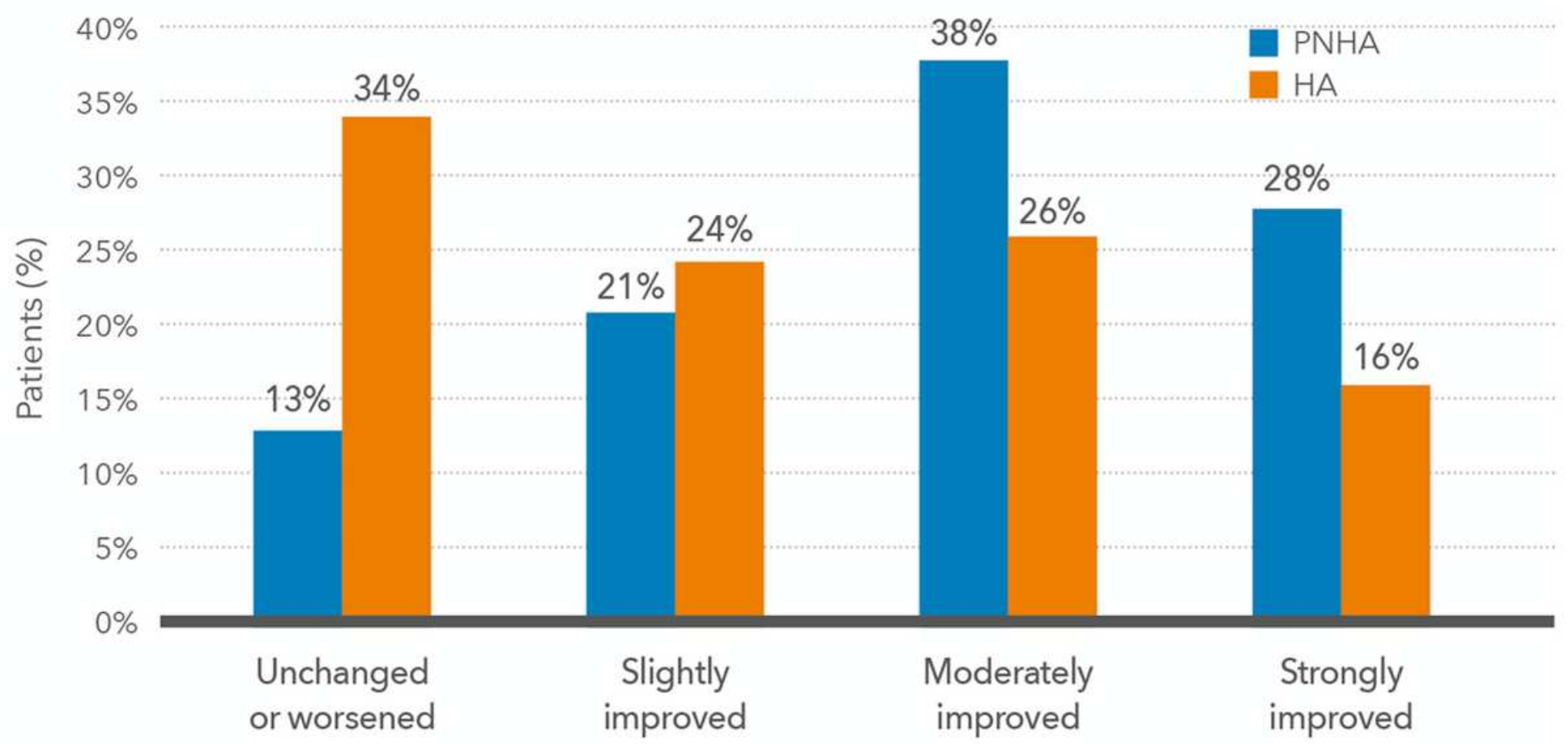

\section{Figure 7}

Percent of patients in the fixed combination (PNHA) and hyaluronic acid (HA) treatment groups reporting improvement in Knee Society Score (KSS) pain scores during the [T3] (2 months) to [T6] (24 months) follow-up period. 


\section{KSS pain scores over the first 2 months of the study}

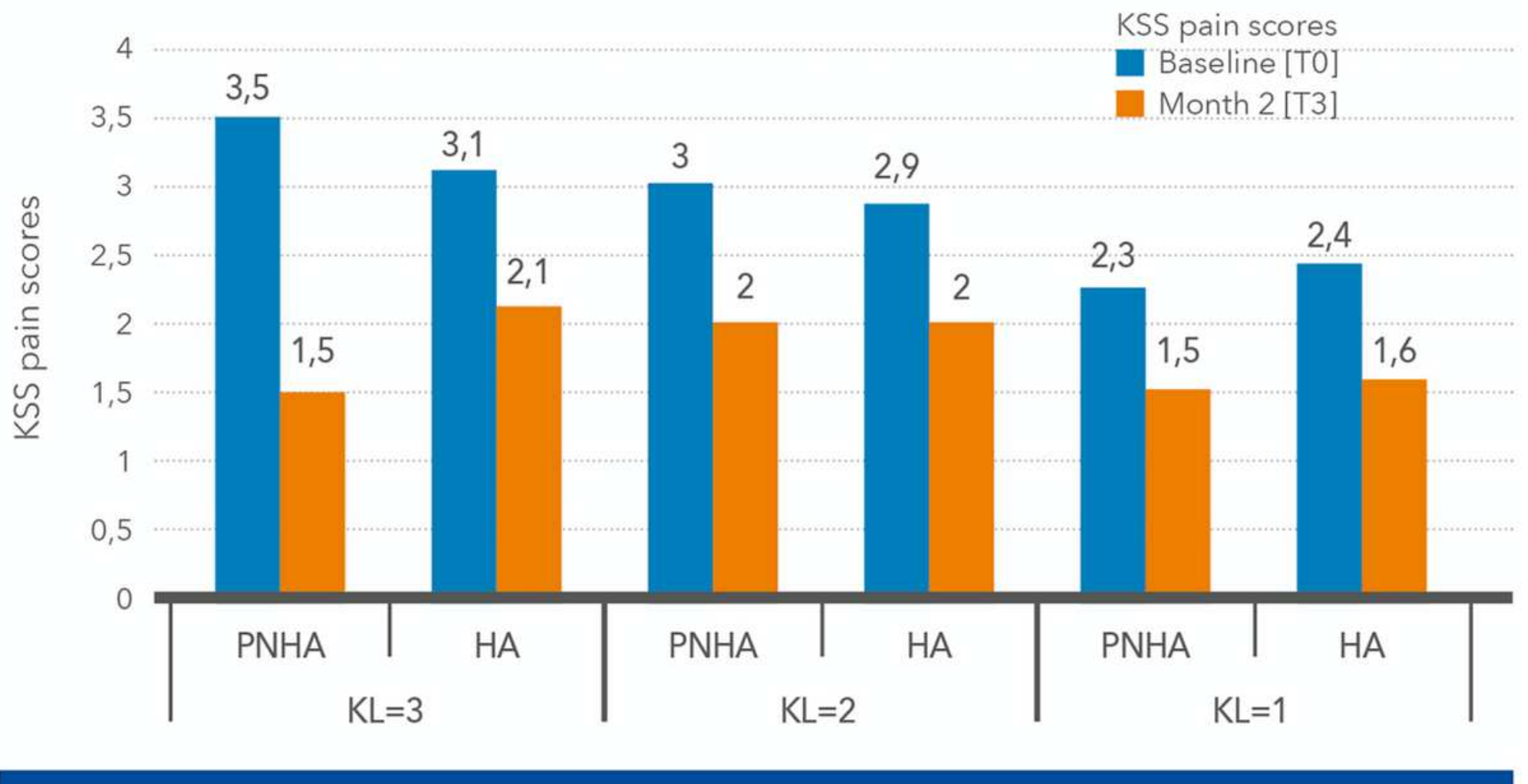

Figure 8

Mean Knee Society Score (KSS) pain scores at baseline and [T3] (2 months) in patients of the fixed combination (PNHA) and hyaluronic acid ( $\mathrm{HA})$ treatment groups according to baseline severity (KellgrenLawrence grade) of knee joint disease. 


\section{Two-year KSS pain scores vs. baseline clinical severity}

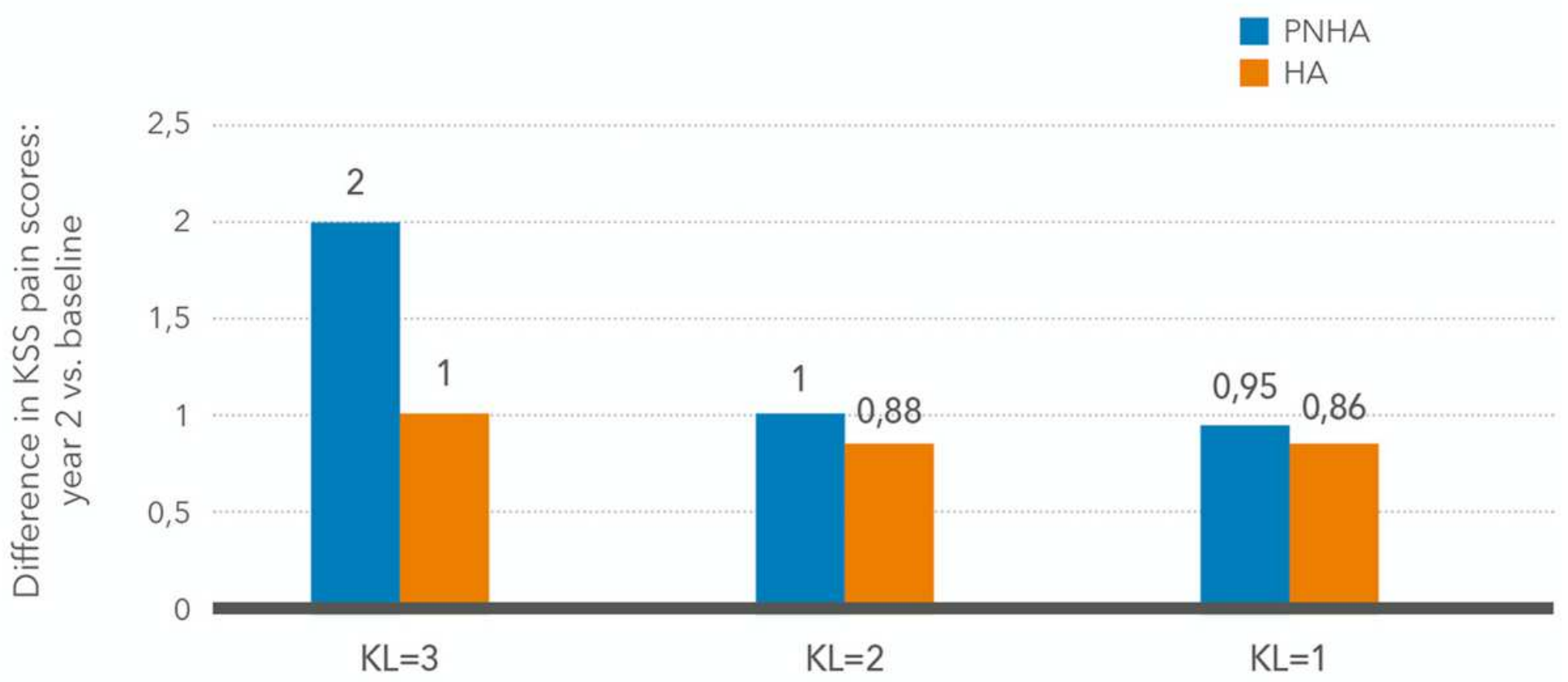

\section{Figure 9}

Improvement in mean Knee Society Score (KSS) pain scores, baseline vs [T6] (24 months) in patients of the fixed combination (PNHA) and hyaluronic acid (HA) treatment groups according to baseline severity (Kellgren-Lawrence grade) of knee joint disease. 


\section{Choosing between PNHA and HA}

\section{Knee OA patients}

KL grade 1-3; grade 4 waiting for TKA

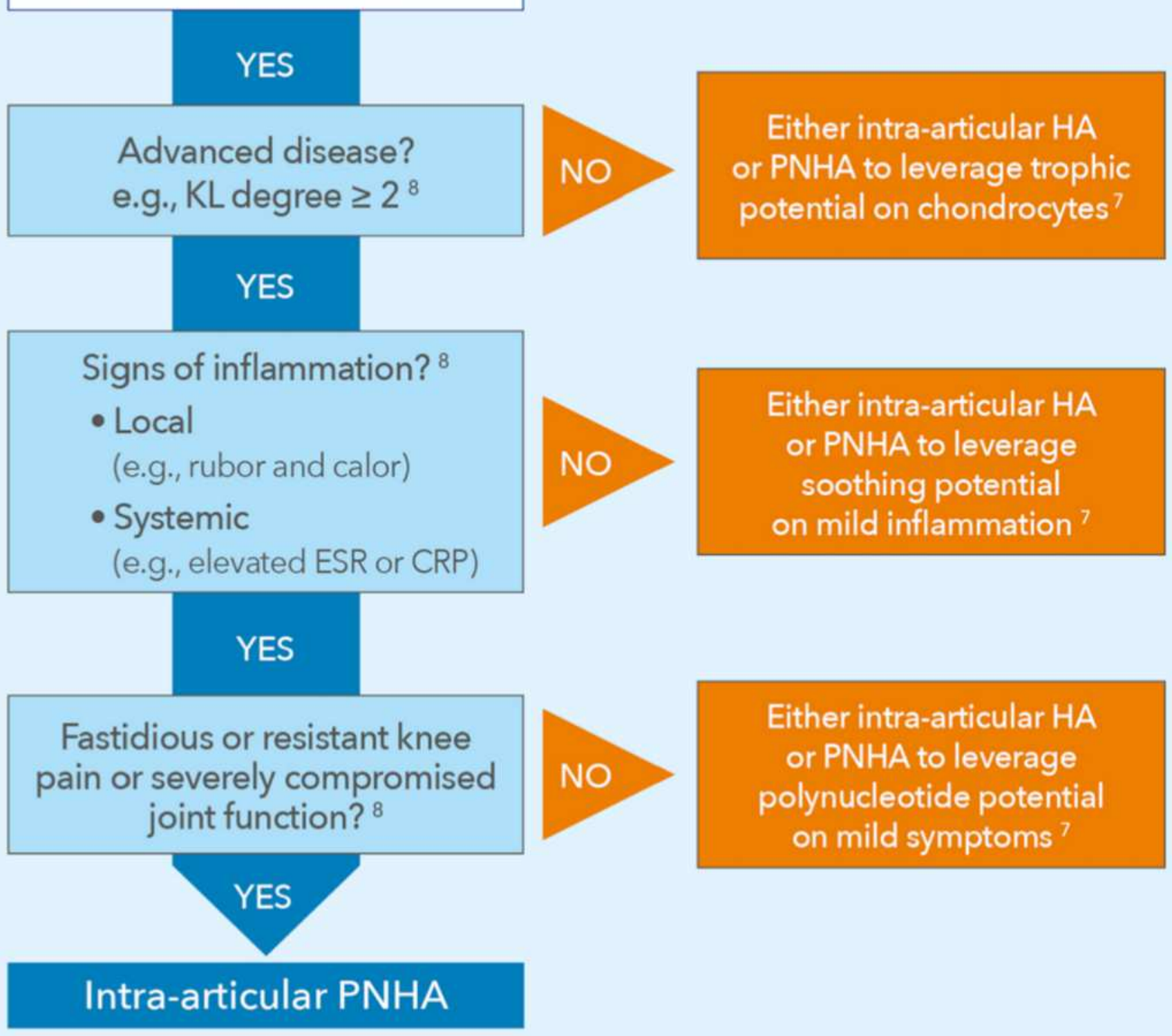

Figure 10

Does an ideal knee OA patient for either PNHA or HA exist? A tentative decisional algorithm.

\section{Supplementary Files}

This is a list of supplementary files associated with this preprint. Click to download. 
- Table1.png

- Table2.png 